\title{
Effective Linewidth in Raman Spectra of Titanium Dioxide Nanocrystals
}

\author{
M. Salis ${ }^{1}$, P.C. Ricci ${ }^{*}, 1$ and A. Anedda ${ }^{1,2}$

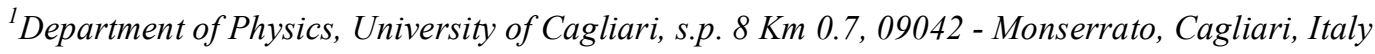 \\ ${ }^{2}$ LIMINA Laboratory, University of Cagliari, s.p. $8 \mathrm{Km}$ 0.7, 09042 - Monserrato, Cagliari, Italy
}

\begin{abstract}
Raman spectra of nanocrystals titanium dioxide are discussed and the correlation between the band shape of the allowed $\mathrm{A}_{1 \mathrm{~g}}$ Raman mode and the crystals dimensions is discussed. Data on Raman spectra are reconsidered in the framework of a modified "hard confinement" model (MHC). The proposed model is based on the idea of using an effective linewidth parameter, which is a function of the effective dimension of the nanostructure, in spite of the intrinsic Raman band linewidth.

The comparison with standard hard confinement model reveals better agreement with the experimental results for the MHC model up to $6 \mathrm{~nm}$. Moreover, the analysis permits to improve the knowledge of the phonon dispersion curve as well as the intrinsic Raman bulk parameters. An analytical form of the size-dependent peak-position in nanocrystals, useful for an approximated size estimation, has been explicated. The general structure of the model permits to extended the MHC to other nano-sized materials.
\end{abstract}

\section{INTRODUCTION}

Agglomerates of nanosized $\mathrm{TiO}_{2}$ crystals are promising materials for electrochemical, photochemical and photovoltaic applications [1-4]. Among the several techniques used to characterize these materials, Raman spectroscopy plays a special role in the investigation of size effects on crystal vibrational properties in the nanometer scale. Depending on the crystal size nanocrystals undergo strain or nonstoichiometry which can significantly affect the Raman spectra [3, 4]. Several reports on titanium dioxide nanocrystals show that Raman shift in this material is mainly due to phonon confinement (PC) effect [1-3]. Different confinement models have been used to fit experiments with alternate successes [1-4], where the main difficulties in matching experiments can be ascribed to model oversimplifications. In this connection, it has been shown that by using the proper model parameters it is possible to greatly improve fits of theoretic Raman spectra to experimental peak-shapes [5]. These results have been obtained by modifying the "hard confinement" (HC) model, where the intrinsic Raman linewidth is replaced by a proper effective one. The latter may be affected by several unpredictable effects such as interactions between defects and band modes [6], changes of the anharmonic coupling factors [7], anisotropy $[8,9]$ and/or phase mixing [3]. Besides, although Raman spectra are surely subject to PC effects, they can show structures significantly scattered from the predictions based on the ideal PC problem. This difference may raise from the proper figures of the effective linewidth parameter utilized in PC models.

*Address correspondence to this author at the Department of Physics, University of Cagliari, s.p. $8 \mathrm{Km}$ 0.7, 09042 - Monserrato, Cagliari, Italy;

E-mail: carlo.ricci@dsf.unica.it
Early and recent data are investigated in this paper. Our goal is to show that for $\mathrm{TiO}_{2}$ nanocrystals the variations in Raman-peak positions due to the occurrence of linewidth broadening should be accounted for by the modified HC (MHC) model. In Section 2 a curve model for the sizedependent effective linewidth will be derived on from the averaged anisotropy function $[8,9]$. This allows an approximated analytical form of the peak-position dependence on particle size that features all the relevant parameters. Deviations of the experimental figures from model predictions are further considered in Section 3.

\section{MODEL SETTINGS}

In a perfect infinite crystal only phonons close to the center of the Brillouin zone (BZ) contribute to inelastic scatterings of incident radiations. When crystal sizes range in the nanometer scale, a larger portion of the $\mathrm{BZ}$ is allowed to effectively participate in scattering processes due to weakening of the rule $q_{0} \approx 0[10,11]$. The extension of such a region in the quasi-momentum space is determined by the confinement of the $q_{0}=0$ phonon wave function $[11,12]$. Formally, this can be explained by considering the modulation of the phonon wave function in the infinite crystal with a suitable "weighting" function [11]. It is possible to calculate the first-order Raman spectrum of nanosized crystals on expansion by Fourier integrals (except for a constant factor) from

$$
I(\omega)=\int_{B Z} \frac{|C(\mathbf{q})|^{2}}{[\omega-\omega(\mathbf{q})]^{2}+\left(\Gamma_{0} / 2\right)^{2}} d \mathbf{q}
$$


where $C(\mathbf{q})$ stands for Fourier coefficients of the $q_{0}=0$ phonon wave function, $\omega(\mathbf{q})$ for the phonon dispersion curve and $\Gamma_{0}$ for the intrinsic Raman band linewidth [11]. Some simplifications are required to handle eq. (1). A drastic but commonly accepted assumption considers an isotropic dispersion in a spherical BZ [11] and the function $\omega(q)$ represents an averaged dispersion curve [13]. As for phonon confinement (PC), the model which received the most credit assumes a Gaussian weighting function (hard confinement, $\mathrm{HC)}$ for spherical nanocrystals $[1,2,8,9,13]$.

Accordingly, eq. (1) can be rewritten as

$$
I(\omega)=4 \pi \int_{0}^{\pi / a} \frac{\exp \left(-q^{2} L^{2} / 16 \pi^{2}\right) q^{2}}{[\omega-\omega(q)]^{2}+\left(\Gamma_{0} / 2\right)^{2}} d q
$$

where $\mathrm{L}$ is the correlation length in the crystal, which can be assumed as the diameter of the nanoparticles, and " $a$ " stands for the lattice parameter.

From the numerical inspection of eq. (2) it can be found that, as long as the quadratic approximation $\omega(q)=\omega_{0}+A q^{2} a^{2} / 2$ holds ( $A$ stands for a suitable curve parameter), the Raman peak-position can be calculated with a good approximation by means of [14].

$\omega_{P}=\omega_{0}+12 \pi^{2} \frac{A a^{2}}{L^{2}}[1-\exp (-L / 12 a x)]$

where $x=\left(|A| / \Gamma_{0}\right)^{1 / 2}$. It is possible to appreciate the effect of the effective linewidth on the peak position by replacing $\Gamma_{0}$ with $\Gamma$ so that

$\partial \omega_{P} / \partial \Gamma=\pi^{2} \frac{a x}{2 L} \exp (-L / 12 a x) \operatorname{sgn} A$

where sgnA stands for the sign of $\mathrm{A}$. The latter equation clearly shows that linewidth can become important in determining the peak position when the size of nanocrystals ranges within a few nanometers (for example, if $L=12 a x$ then $\left.\left|\partial \omega_{P} / \partial \Gamma\right|=0.41\right)$.

Previous works propose to replace the effective linewidth $\Gamma_{0}$ with a function $\Gamma^{2}(q)=\Gamma_{0}^{2}+\gamma(q)^{2}$, where $\gamma(q)$ was referred to as the anisotropy correction which accounts for the spreading of the phonon band over the BZ to explain the anomalous broadening of Raman peak-width. This correction is defined as twice the root mean square deviations of the actual $\omega_{i}(q)$ from the average $\bar{\omega}(q)$ [8,9]. However, there may be other reasons for dealing with a function $\Gamma(q)$. For example, in bulk materials quasi-stationary modes originated by interactions between defect and band modes enlarge the frequency distribution around unperturbed modes with resonance width depending on quasi-momentum, thus affecting relaxation time in phonon-photon (or -particle) interactions [6]. The large defect disorder, which is possible in high energy state crystals, may smear the broadening over all the band modes. However, inspection of the Raman peak shapes suggests that $\Gamma_{0}$ can actually be replaced by a parameter $\Gamma$ which partially depends on crystal size [5]. Thus, rather than a function $\Gamma(q)$, we may consider its average over the phonon wave function.

In order to account for quasi-momentum dependence in $\Gamma(q)$ we propose to consider the function as

$$
\Gamma^{2}(q)=\Gamma_{0}^{2}+\lambda^{2}(q a)^{4}
$$

which presents the same analytical structure as $\gamma(q)$ until phonon curves in the anisotropic BZ are considered in the quadratic approximation.

The working hypothesis is

$$
\Gamma^{2}(L)=\int_{0}^{\pi / a}|C(\mathbf{q}, L)|^{2} \Gamma^{2}(q) d \mathbf{q} / \int_{0}^{\pi / a}|C(\mathbf{q}, L)|^{2} d \mathbf{q}
$$

where the dependence on crystal size of Fourier coefficients has been explicated.

When crystal size is sufficiently larger than crystal parameter $a$, it is possible to calculate the integrals in eq. (6) extending the upper limit to infinity:

$\Gamma^{2}(L)=\Gamma_{0}^{2}+\left[30.9 \frac{\pi^{2} a^{2}}{L^{2}} \lambda\right]^{2}$

where is to be estimated from comparison with experiments and $\Gamma^{2}(L)$ itself integrates all the effects contributing to the enlargement of the effective linewidth. It is worth to note that eq. (7) itself represents an assumption that could only roughly estimate the function dealt with.

\section{RESULTS AND DISCUSSION}

In Fig. (1) data on peak-widths are reported from refs. [1, $2,5]$. The peak-widths calculated from the MHC model by using eq. (7) in eq(2) are reported as well (solid curve). According to refs $[1,2,5]$, the dispersion curve used for the calculations in case of Anatase phase is:

$\omega(q)=\omega_{0}+A[1-\cos (q a)]$

with $\omega_{0}=144 \mathrm{~cm}^{-1}, A=20 \mathrm{~cm}^{-1}$ and $a=0.376 \mathrm{~nm}$; for the curve $\Gamma(L)$ we used $\Gamma_{0}=7 \mathrm{~cm}^{-1}[1,2,5]$ and $\lambda=15 \mathrm{~cm}^{-1}$. It is to be pointed out that parameter $\lambda$ is established by fitting peak-width predictions to experimental data in the range $L<16 \mathrm{~nm}$. With this choice, large departures from experiments in the upper size range can be observed, but here $\partial \omega_{p} / \partial \Gamma$ is small and the peak-position is weakly affected by peak-width.

Peak positions were calculated by using the "anatase" values": and the results are shown in Fig. (2, black solid curve obtained from eq. 2) in which $\Gamma_{0}$ is replaced by 
$\Gamma(L))$ where, by comparison, the curve predicted by the non-modified HC model is reported as well (blue solid curve obtained from eq. 2).

The curves obtained from the two model are very similar at large nanoparticle dimensions (about $12 \mathrm{~nm}$ ), while sensible differences appear where phonon confinement effect plays a major role. In this range the MHC model fits the experimental result much better than the HC model (Fig. 2). However, it is worth pointing out that both the models do not fit adequately the experimental data for particles of large dimensions, where the choice of the intrinsic Raman bulk parameters and the phonon dispersion curve are critical. Therefore the fit has been performed with small variation in the phonon dispersion curve parameters (eq. 8); the best result has been obtained for: $\omega_{0}=143.5 \mathrm{~cm}^{-1}$, with $A=23 \mathrm{~cm}^{-1}$ and the parameter $\lambda$ of eq. 7 becomes $\lambda=13.8 \mathrm{~cm}^{-1}$ (red solid curve). The obtained value for the intrinsic Raman frequency of the $A_{1 g}$ Raman mode is still under the spectral resolution of the experimental data. With respect to the previous case we obtained a better fit in the large size range also for the $\mathrm{HC}$ model, but we also observed that it failed in the low nanoparticle diameter range. The peak-widths calculated from the MHC model are not affected by these variation (Fig. 1).

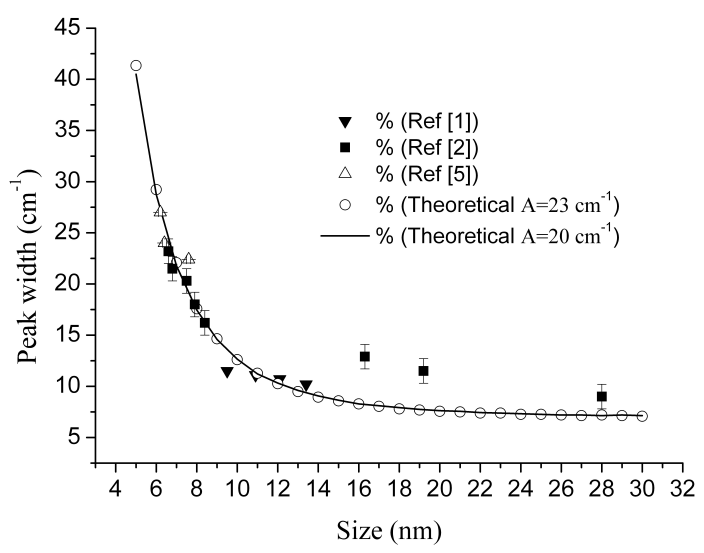

Fig. (1). Experimental and theoretical peak-widths. The latter were calculated by replacing the intrinsic linewidth with the effective one defined in eq. (7).

From comparison of data shown in Fig. (2) we note the presence of some discrepancies between model predictions and experiments that cannot be explained by linewidth broadening. Thus, to go deeper into this analysis, it is convenient to make an estimation, even though rough, of the behaviour of $A$ (to avoid confusion, it will be referred to as $A_{R}$ ) as a function of the nanoparticle dimension. This can be made by taking into account that when peak-width is large the peak shape features an almost Lorentz-shaped. Thus, we retrieved the searched-for parameters by fitting the MHC model to the Lorentz-like curves having the actual Raman peak-widths and -positions. This was done by using a standard Levenberg-Marquardt procedure [5, 15]. As for data in ref [5] we are able to report the fit results of the actual Raman curves. To simplify the discussion all the retrieved pa- rameters are obtained only for the case $\omega_{0}=143.5 \mathrm{~cm}^{-1}$ where the fitting curve for both models gives the best results. Table 1 reports the parameters calculated for different nanoparticle dimensions: $\bar{\omega}$ stands for the actual peak position, $\widehat{\Gamma}$ for the actual peak width, $\Gamma$ and $A_{R}$ for the retrieved effective linewidth and phonon curve parameter (eq. 8 is used in eq. 2), respectively.

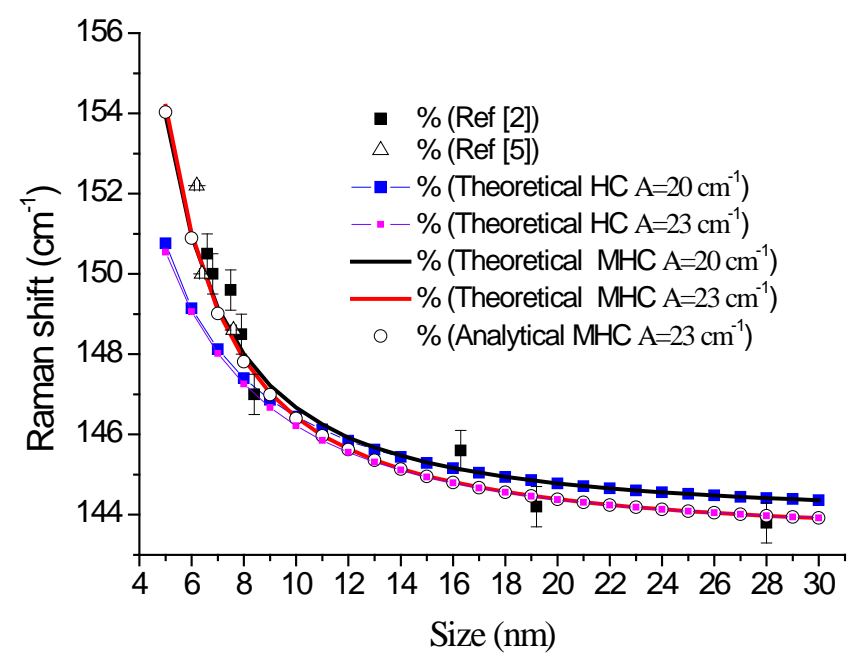

Fig. (2). Experimental and theoretical peak-positions. The latter were calculated by replacing the intrinsic linewidth with the effective one defined in eq. (7). Circles show the curve calculated by means of the analytical form (3) and eq. (7).

It is worth pointing out that in spite of the effective linewidths, which appear to increase almost monotonically as crystal size decreases, parameter $A_{R}$ shows a non-regular behaviour dependent on the actual sample. However, its value oscillates, sample by sample, around which is the expected value from the chosen $\omega_{0}$. The results suggest that the mechanism determining the $\Gamma$ broadening and $A_{R}$ deviations are poorly correlated, being the former strongly connected to the effective nanoparticle dimension, while the latter $\left(A_{R}\right)$ to the phonon dispersion curve. Finally, on the basis of the previous results we are able to give an analytical form of the peak-position dependence on particle size. Indeed, from eq. (3) with $\omega_{0}=143.5 \mathrm{~cm}^{-1}, A=23 \mathrm{~cm}^{-1}$ and $x=\sqrt{A / \Gamma(L)}$ with $\lambda=13.8 \mathrm{~cm}^{-1}$ we obtain a curve (circles) very close to the one predicted by MHC model. The choice of giving an analytical form of $\omega_{P}(L)$ to titanium dioxide nanocrystals may be useful in characterizations of samples grown by means of properly standardized preparation methods. In particular, in the perspective of industrial applications, it could allow an approximated size estimation based on few and easily obtained experimental data.

Besides, the results show light deviation from sample to sample, and this work points out that an effective linewidth parameter, which is function of the dimension of the nanostructure, should be considered for a correct interpretation of nanoparticle Raman spectra. The model has been applied 
Table 1. Actual Crystal Size L, (Mean) Peak-Position $\omega$ and -Width $\Gamma$ Considered in Figs. (1, 2). $\Gamma$ and $A_{R}$ Stand for the Retrieved Effective Linewidth and Phonon Curve Parameters Obtained by Using a Standard Levenberg-Marquardt Fitting Procedure

\begin{tabular}{|c|c|c|c|c|}
\hline$L(\mathrm{~nm})$ & $\widehat{\omega}\left(\mathrm{cm}^{-1}\right)$ & $\hat{\Gamma}\left(\mathrm{cm}^{-1}\right)$ & $\Gamma\left(\mathrm{cm}^{-1}\right)$ & $A_{R}\left(\mathrm{~cm}^{-1}\right)$ \\
\hline 28 & 143.8 & 9 & 9 & 23.3 \\
\hline 19.2 & 144.2 & 11.5 & 11.5 & 21.3 \\
\hline 16.3 & 145.6 & 12.9 & 12.5 & 34 \\
\hline 8.4 & 147 & 16.2 & 14.5 & 18 \\
\hline 7.9 & 148.4 & 18 & 15.7 & 23.3 \\
\hline $7.6^{*}$ & 148.6 & 22.4 & 18.5 & 22.5 \\
\hline 7.5 & 149.6 & 20.3 & 17 & 27 \\
\hline 6.8 & 150 & 21.5 & 18 & 23.5 \\
\hline 6.6 & 150.4 & 23.2 & 19.3 & 24 \\
\hline $6.4 *$ & 150 & 24 & 19 & 20.8 \\
\hline $6.2 *$ & 152.2 & 27 & $20.5 *$ & 26.6 \\
\hline
\end{tabular}

only on titanium dioxide nanocrystals but can be extended to other nano-sized materials. Further analysis on other materials are a mandatory task.

\section{CONCLUSIONS}

A modified hard confinement model has been proposed. We have shown that for a better matching of the $\mathrm{HC}$ model with Raman spectra of nanocrystals a suitable definition of an effective linewidth is required. The model is focused on the replacement of the intrinsic Raman linewidth in the $\mathrm{HC}$ model with a function of the actual dimension of the nanostructure. The model has been successfully applied to titanium dioxide nanocrystals and further information about the phonon dispersion curve has been obtained. An analytical form of the developed model, useful for a approximated size estimation, has been explicated.

\section{REFERENCES}

[1] Bersani D, Lottici PP, Ding XZ. Phonon confinement effects in the Raman scattering by $\mathrm{TiO}_{2}$ nanocrystals. Appl Phys Lett 1999; 72: 73-5.

[2] Zhang F, He YL, Zhang MS, Yin Z, Chen Q. Raman scattering study on anatase $\mathrm{TiO}_{2}$ crystals. J Phys D: Appl Phys 2000; 33: 912-6.

[3] Swamy V, Kuznetsov A, Dubrovinsky LS, Caruso RA, Shchukin DG, Muddle BC. Finite-size and pressure effects on the Raman spectrum of nanocrystalline anatase $\mathrm{TiO}_{2}$. Phys Rev B 2005; 71: 184302.
[4] Mazza T, Barborini E, Piseri P, et al. Raman spectroscopy characterization of $\mathrm{TiO}_{2}$ rutile nanocrystals. Phys Rev B 2007; 75: 045416.

[5] Ricci PC, Salis M, Anedda A. Phonon characterization of nanocrystals by Raman spectroscopy. Chem Phys Lett 2008; 457: 1913.

[6] Maradudin AA, Lifshitz IM, Kosevich AM, Cochran W, Musgrave MJP. Lattice Dynamics. W. A. Benjamin, Inc.: New York 1969.

[7] Ursaki VV, Tiginyanu IM, Ricci PC, Anedda A, Foca EV, Syrbu NN. Temperature dependence of Raman scattering in porous gallium phosphide. J Phys Condens Matter 2001; 13: 4579- 89.

[8] Carles R, Mlayah A, Amjoud M, Reynes A, Morancho R. Structural characterization of Ge Microcrystals in $G e_{x} C_{1-x}$ Films. Jpn J Appl Phys 1992; 31: 3511-14.

[9] Ricci PC, Casu A, De Giudici G, Scardi P, Anedda A. Phonon confinement effect in calcium fluoride nanoparticles. Chem Phys Lett 2007; 444: 135-38.

[10] Richter H, Wang ZP, Ley L. The one phonon Raman spectrum in microcrystalline silicon. Solid State Comm 1981; 39: 625- 9.

[11] Campbell IH, Fauchet PM. The effects of microcrystal size and shape on the one phonon Raman spectra of crystalline semiconductors. Solid State Comm 1986; 58: 739-41.

[12] Faraci G, Gibilisco S, Russo P, Pennisi AR, La Rosa S. Modified Raman confinement model for Si nanocrystals. Phys Rev B 2006; 73: 033307.

[13] Dieguez A, Romano-Rodriguez A, Vilà A, Morante JR. The complete Raman spectrum of nanometric $\mathrm{SnO}_{2}$ nanoparticle. J App Phys 2001; 90: 1550-57.

[14] Salis M, Ricci PC, Anedda A. An analytical form for the Raman shift dependence on size of nanocrystals. J Raman Spectrosc 2008; 40: 64-66.

[15] Press WH, Teukolsky SA, Vetterling WT, Flannery BP. Numerical recipes in Fortran. Cambridge University Press: Cambridge 1992. 